\title{
Síntesis de Hidrogeles de Acrilamida en Soluciones Acuosas de Etanol
}

\author{
Luis Guillermo de Jesús García Sánchez, Jorge Alberto Cortés Ortega \\ Departamento de Química, Universidad de Guadalajara, México
}

Resumen: En el presente trabajo se realizó la polimerización de acrilamida en soluciones acuosas de etanol, variando la composición del etanol en la solución; obteniéndose tanto nanogeles como macrogeles. Se determinó la capacidad de absorber agua de los macrogeles; obteniéndose materiales que tienen una capacidad de absorber desde 40 y hasta 90 gramos de agua por gramo de xerogel y el tamaño de partícula de los nanogeles fueron desde $71 \mathrm{~nm}$ y hasta $463 \mathrm{~nm}$.

Palabras clave: Acrilamida, etanol, nanoparticulas.

\section{Polymerization of Acrylamide Hydrogels in Aqueous Ethanol}

\begin{abstract}
The polymerization of acrylamide was performed in aqueous ethanol solutions, varying the concentration of ethanol in the solution; obtaining both nanogels as macrogels. The water absorbing capacity of the macrogels were found; obtaining materials with a capacity to absorb from 40 to $90 \mathrm{~g}$ of water per gram of xerogel and the particle size of the nanogels are at values ranging from $71 \mathrm{~nm}$ to $463 \mathrm{~nm}$.
\end{abstract}

Keywords: Acrylamide, etanol, nanoparticles.

\section{Introducción}

Incrementar la capacidad de absorber agua en los hidrogeles se ha convertido en tema de estudio en los últimos años. Los hidrogeles son redes poliméricas que se hinchan considerablemente en presencia de agua manteniendo su forma hasta alcanzar un equilibrio fisicoquímico ${ }^{[1]}$. El carácter hidrófilo de estos materiales, se debe a la presencia de grupos compatibles con el agua, tales como $-\mathrm{OH},-\mathrm{COOH},-\mathrm{CONH} 2,-\mathrm{SO} 3 \mathrm{H}$. Por otro lado, en estado deshidratado, un hidrogel se denomina xerogel y tiene estructura cristalina ${ }^{[2]}$. Las propiedades de los hidrogeles, permiten su utilización en diferentes campos $^{[3,4]}$. Entre las propiedades de los hidrogeles, la temperatura de transición de volumen de fase (TTPV), ha atraído gran atención desde su descubrimiento ${ }^{[5,6]}$. El estudio de la transición de volumen de fase fue iniciado por la predicción teórica de Dusek y Patterson ${ }^{[7,8]}$. Estos autores sugirieron la posibilidad del cambio discontinuo de volumen de un gel (TTPV), basándose en la analogía de la transición de ovillo-glóbulo ("coil-globule") de polímeros en solución, la cual fue predicha por Pitsyn ${ }^{[9]}$. La transición de fase en volumen fue descubierta experimentalmente en 1978 por Tanaka ${ }^{[10]}$, en un gel parcialmente ionizado de poliacrilamida en una mezcla de acetona-agua. Esta transición de fase se observó no sólo al cambiar la composición del solvente, sino también al variar la temperatura, la concentración de iones, el pH o el campo eléctrico. Se ha encontrado que los geles de poliacrilamida poli(AM) no absorben etanol, sin embargo el monómero acrilamida es soluble en soluciones acuosas de etanol e inclusive en etanol ${ }^{[11]}$. Por lo que se puede esperar que hidrogeles preparados con este monómero presenten esta transición al incrementar la concentración de etanol en la solución. La capacidad de absorción de los hidrogeles depende (como se establecio anteriormente), de las características químicas y físicas de los monómeros utilizados en su síntesis; se puede incrementar la capacidad de absorber agua si se crean suficientes huecos dentro de la red polimérica, para permitir la presencia de agua no $\operatorname{asociada}^{[12]}$, por lo que un hidrogel no homogéneo tendrá una mayor capacidad de absorber agua, materiales con estas características se han sintetizado en el pasado, adicionando partículas nanométricas sintetizadas mediante reacción de polimerización tanto en emulsión o microemulsión ${ }^{[13,14]}$. En estas aplicaciones el uso de tensoactivos para la formación de las partículas es necesario, con lo que las partículas y/o los hidrogeles se tienen que someter a procesos de purificación y limpieza. En otros métodos se han sintetizados hidrogeles macroporosos mediante la adición de componentes que se gasifique o se descompongan en sustancias gaseosas inertes, cuando ocurra la reacción de polimerización, contribuyendo a la creación de huecos dentro de la red ${ }^{[15,16]}$; por lo que la búsqueda de sintetizar hidrogeles macroporosos que incrementen la capacidad de absorber agua, sin tener que utilizar aditamentos que puedan incrementar los procesos de purificación o contaminación, con lo cual se elevan tanto los costos de producción así como el material contaminante residual, se convierte en un campo de interés a desarrollar.

\section{Métodos y Materiales}

Acrilamida (AM) con una pureza de 99\%, grado electroforético, de Aldrich chemical company. Agua bidestilada, desionizada, con un $\mathrm{pH}$ de 6.75, de Productos Selectropura, S.A. de C.V. El iniciador empleado fue Peroxidisulfato de Potasio (KPS con una pureza de 99\%) 
de Alfa Aesar. Como agente entrecruzante se utilizó N, N-metilenbisacrilamida, (NMBA). El acelerador utilizado fue NNNN-Tetrametil-etilendiamina (TMDA) ambos de TCI Tokio Kasei.

\section{Síntesis del hidrogel convencional $(H C)$}

Se preparo una solución acuosa al 5\% de acrilamida que contiene $1 \%$ de agente entrecruzante con respecto del monómero, una vez formada la solución se adiciono $1 \%$ de iniciador y $1 \%$ acelerador con respecto de la acrilamida, dejándose reaccionar por 1 día a $25^{\circ} \mathrm{C}$. La reacción se lleva a cabo en recipientes cilíndricos de vidrio. (todos los \% son en masa)

\section{Síntesis de poliacrilamida en soluciones acuosas de etanol (HE)}

Posteriormente se prepararon soluciones acuosas de etanol, variando la proporción de etanol en las soluciones, en las cuales se llevaron a cabo las reacciones de polimerización de acrilamida, conservando la misma proporción de monómero/solvente que el hidrogel convencional (5 gramos de monómero/95 gramos de solución), así como la proporción de agente entrecruzante, iniciador y acelerador. Dejándose reaccionar por 1 día.

\section{Medición del tamaño de partícula}

Para la determinación del tamaño de partícula se utilizó el dispersor cuasielastica de luz marca Zetasizer NANO ZS90, el material seco obtenido de las reacciones de polimerización, fue redispersado en agua para determinar el tamaño de partícula, para cada muestra se realizaron tres mediciones, reportándose el promedio del tamaño de partícula.

\section{Análisis morfológico de los hidrogeles sintetizados}

La preparación de la muestras para ser observadas bajo el microscopio electrónico de barrido Hitachi TM-100 fue: se colocan los macrogeles a hinchar en agua bidestilada y cuando alcanzan el hinchamiento de equilibrio, se colocan en etanol absoluto, al cabo de tres días la muestras han colapsado (Figura 1) adquiriendo un color blanco lechoso y se procede al análisis microscópico.

\section{Proceso de limpieza (macrogeles)}

Transcurrido el tiempo de reacción, se remueven los hidrogeles del recipiente y se colocan a secar, pesándolos constantemente hasta que el peso no cambie (xerogel), una vez secos se colocan en agua destilada, a $25^{\circ} \mathrm{C}$ por una semana, para permitir que las muestras alcancen el hinchamiento de equilibrio, con lo cual tanto el monómero residual como los oligómeros no adicionados a la red son desalojados, al termino de este tiempo se cambia el agua de lavado y se dejan por dos días en agitación constante, al termino de este tiempo se les cambia el agua de lavado, este proceso se repite en tres ocasiones. Con el peso final e inicial, se determina el rendimiento de la reacción de polimerización.

\section{Cinética de hinchamiento}

Las muestras totalmente secas (macrogeles), se pesan y se colocaron en agua bidestilada a $25^{\circ} \mathrm{C}$ para determinar su cinética de hinchamiento. Esto se realizo pesando las muestras a diferentes tiempos, secando su superficie con papel absorbente. De la diferencia entre el peso de la muestra seca y el peso de la muestra hinchada se calcula el hinchamiento con la Ecuación 1.

$$
\mathbf{H}=\frac{\text { peso } \mathbf{h n m e d o}-\text { peso seco }}{\text { peso seco }}
$$

Al graficar el hinchamiento contra el tiempo se obtiene la cinética de hinchamiento (Figura 1).

\section{Resultados y Discusiones}

Se obtuvieron macrogeles cuando la reacción de polimerización se realizo en las soluciones que contienen hasta el $15 \%$ de etanol. Estos materiales presentaron una mayor capacidad de absorber agua que los hidrogeles convencionales (Figura 1). La poliacrilamida entrecruzada no absorbe etanol, por lo que durante el crecimiento de las cadenas poliméricas y la formación de la red, el alcohol contenido en la solución, se comienza agrupar en diferentes zonas, impidiendo que las cadenas en crecimiento puedan atravesar dichos espacios, con lo que se crean huecos dentro de la red, obteniéndose un material heterogéneo; que permite la presencia de mayor cantidad de agua no asociada dentro de la red. Se ha establecido que el contenido de agua dentro del hidrogel se debe a interacciones con la red polimérica (agua asociada) y el agua que ocupa los espacios vacíos entre la cadenas de polímero (agua no asociada). Un indicio de la formación de los huecos se puede observar al comparar el tamaño de los xerogeles obtenidos después del proceso de secado, cuando se introducen en etanol absoluto se puede observar que el tamaño del hidrogel convencional

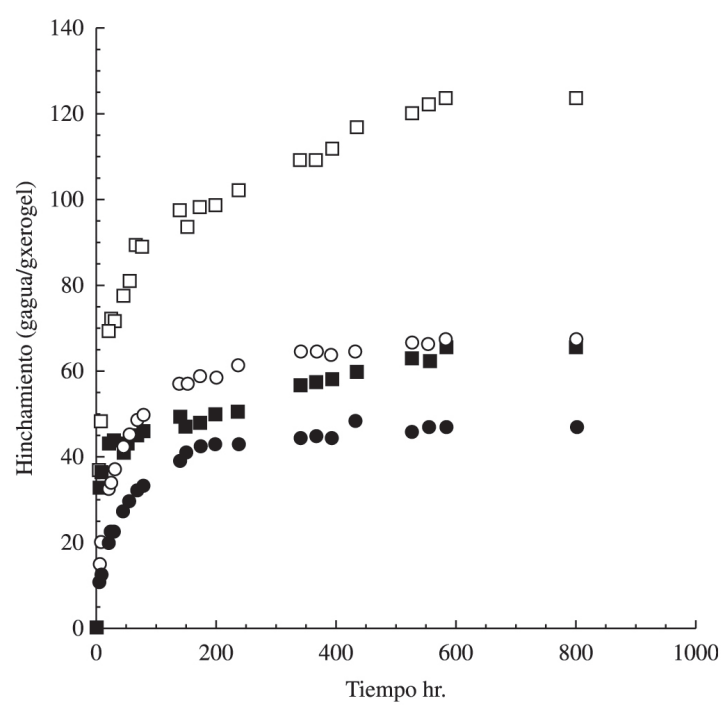

Figura 1. Cinética de hinchamiento de los macrogeles de acrilamida como una función de la concentración de etanol en la solución de pregel. $0 \%(\bullet), 5 \%$ (•), 10\% (०) y 15\% (口). 
disminuye solo un poco, en tanto que los hidrogeles sintetizados en solución de etanol cambian drásticamente su tamaño y cuando se secan en aire, se puede observar que a medida que la proporción de etanol en la mezcla inicial de reacción se incrementa, el xerogel obtenido forma de una película delgada semitransparente, perdiendo su forma cilíndrica obtenida durante la síntesis (Figura 2).

Así mismo se puede observar en las fotos tomadas con el microscopio de barrido (Figura 3) que en los hidrogeles sintetizados en solución acuosa de etanol, se observan las arrugas que se forman cuando se desecan en etanol absoluto. En los experimentos realizados para determinar la reversibilidad en el hinchamiento de las muestras se pudo observar que la muestra $85 / 15$ presentaba una perdida gradual de material en la segunda y tercera ocasión en que se coloca en agua bidestilada, por otro lado las masas de las otras muestras se mantenía constante, así como su capacidad de absorber agua (Tabla 1).

En tanto que el resto de las muestras no solidifican. Como se ha establecido anteriormente la acrilamida es soluble en etanol y en soluciones acuosas de etanol, pero el polímero entrecruzado no es capaz de absorber etanol, por lo que cuando la reacción de polimerización comienza y las partículas están en crecimiento, se forman dos tipos de zonas
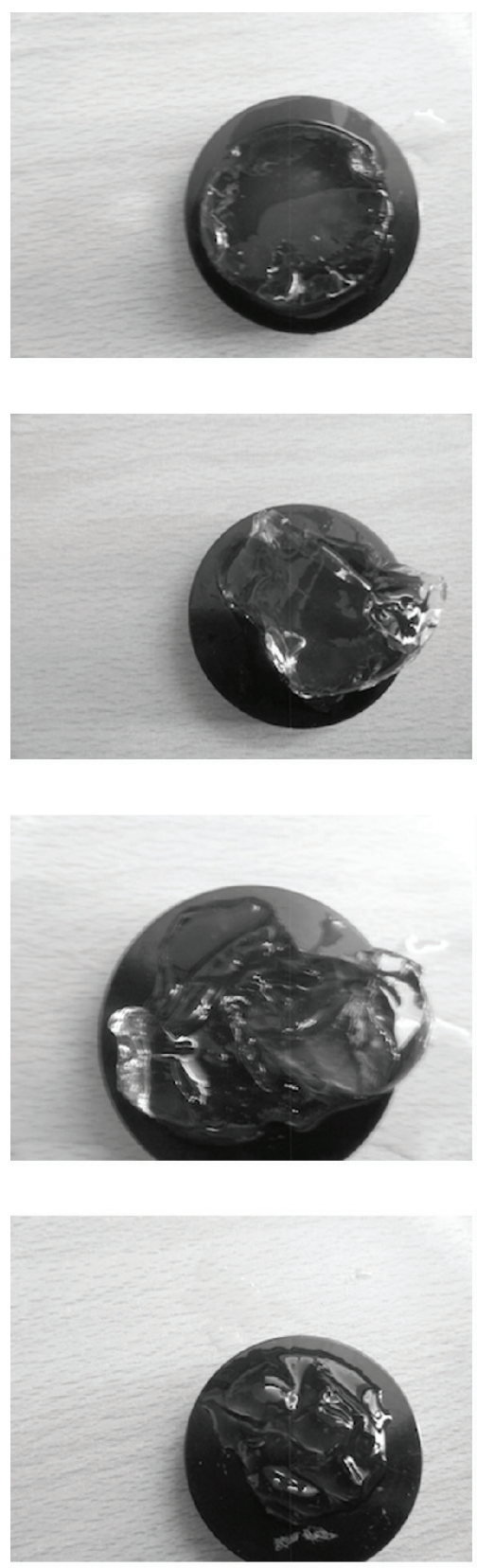

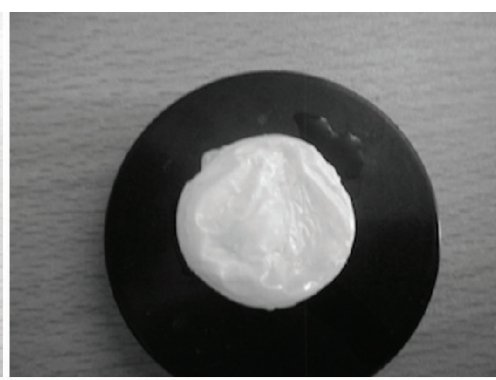

(a)

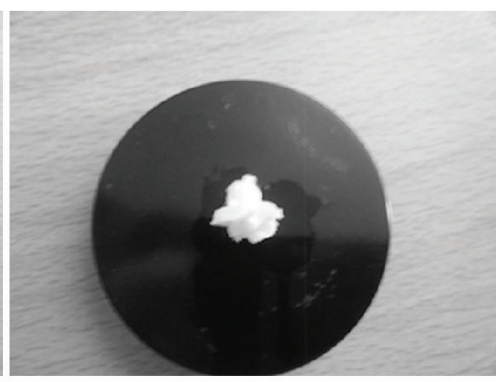

(b)

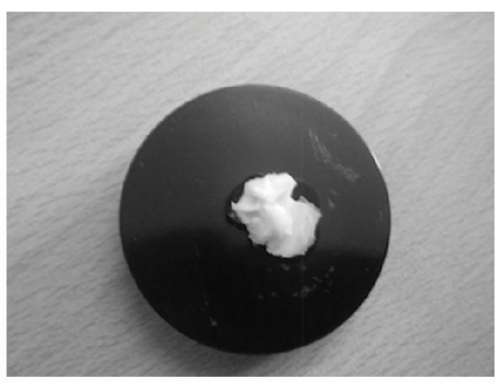

(c)
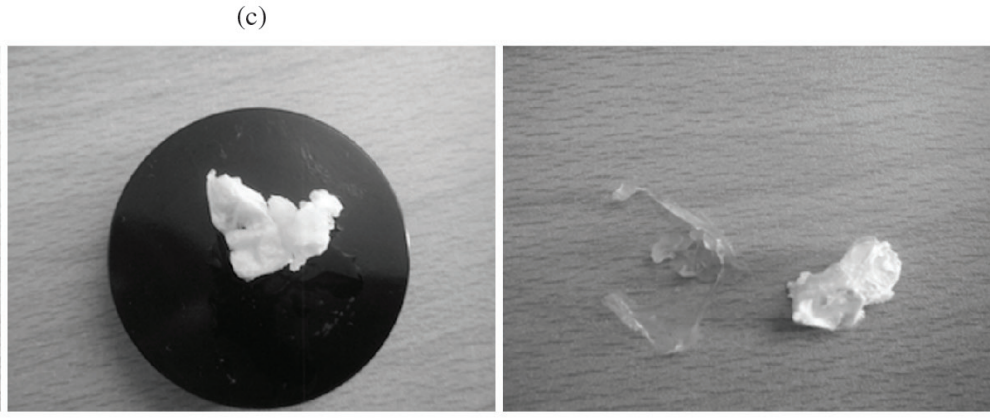

(d)
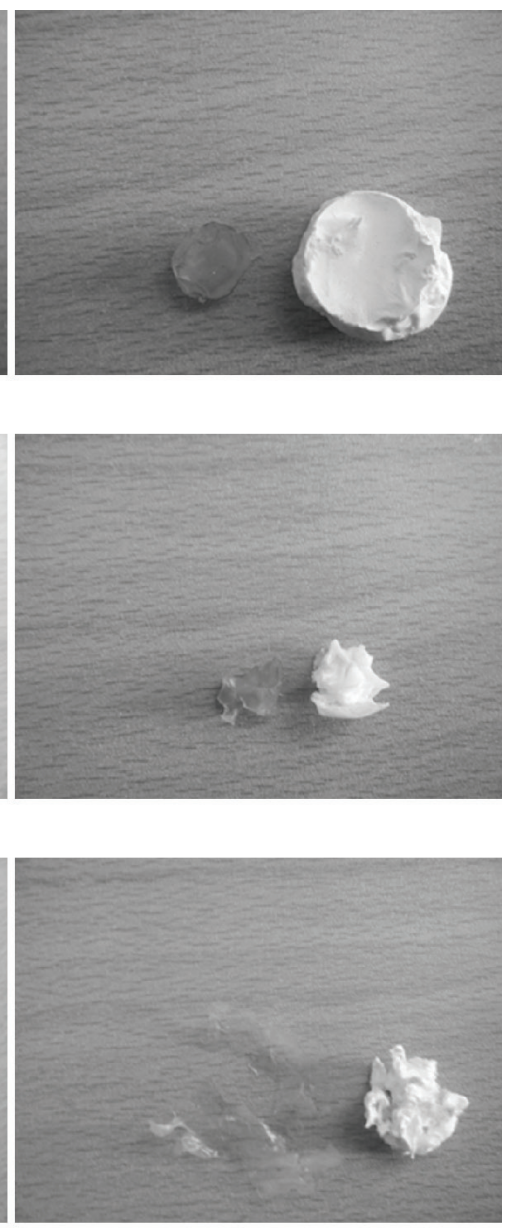

Figura 2. Apariencia física de los macrogeles, en el estado hinchado, cuando son desecados en etanol absoluto y cuando se colocan a secar en el medio ambiente en función de la composición de etanol en la solución de pregel, a) 100/0, b) 95/5, c) 90/10 d) 85/15. 


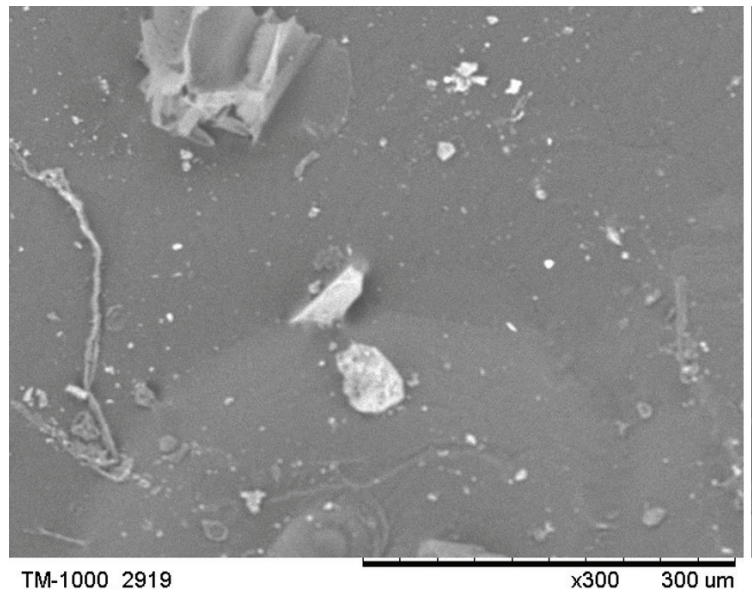

(a)

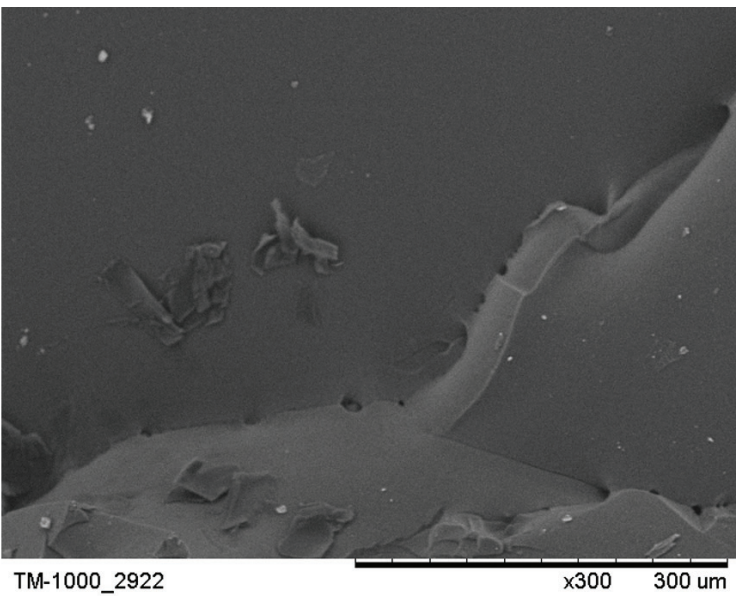

(b)

Figura 3. Micrografía de los macrogeles en función de la relación agua/etanol, 100/0 (a) y 95/5 (b).

Tabla 1. Capacidad de absorber agua, reversibilidad en la capacidad de absorber agua, conversión de la reacción de polimerización e hinchamiento de equilibrio.

\begin{tabular}{|c|c|c|c|c|}
\hline Agua/etanol (masa) & $100 / 0$ & $95 / 5$ & $90 / 10$ & $85 / 15$ \\
\hline Conversión & $98 \pm 0.2$ & $97 \pm 0.8$ & $92 \pm 0.5$ & $90 \pm 1.0$ \\
\hline Hinchamiento $\mathrm{g}_{\text {agua }} / \mathrm{g}_{\text {xerogel }}$ & $49.157 \pm 2$ & $69.414 \pm 3$ & $64.290 \pm 2$ & $124.340 \pm 3$ \\
\hline Segunda ocasión & 47.188 & 70.481 & 65.484 & 87.956 \\
\hline Tercera ocasión & 49.1234 & 68.938 & 65.232 & 90.443 \\
\hline
\end{tabular}

Tabla 2. Tamaño de partícula de los nanogeles.

\begin{tabular}{lccccc}
\hline Agua/etanol (masa) & $\mathbf{8 0} / \mathbf{2 0}$ & $\mathbf{6 0} / \mathbf{4 0}$ & $\mathbf{5 0 / 5 0}$ & $\mathbf{4 0 / 6 0}$ & $\mathbf{2 0 / 8 0}$ \\
\hline Tamaño de partícula (nm) & $71 \pm 5$ & $121 \pm 4$ & $213 \pm 8$ & $282 \pm 11$ & $463 \pm 12$ \\
\hline
\end{tabular}

dentro de la mezcla de reacción, una rica en agua donde las cadenas del polímero es capaz de crecer formando las partículas (por esta razón se pueden formar los macrogeles cuando la composición de etanol es baja) y la otra rica en etanol por donde las cadenas de polímero ven limitado su crecimiento y esto evita la formación de la macrored. Por lo que las partículas en crecimiento se rodean por capas de solución acuosa de etanol, lo que permiten la estabilidad de las partículas en crecimiento, diversos autores han reportado este fenómeno cuando realizan la polimerización de monómeros y sus polímeros insolubles en agua, por ejemplo cuando sintetizan el polimetacrilato, se adicionan pequeñas cantidades de polivinil alcohol que estabiliza las partículas en crecimiento ${ }^{[16]}$, con lo cual se establece un sistema coloidal; para nuestro caso la solución acuosa de etanol estabiliza las partículas en crecimiento, funcionando como la fase continua, semejante a un sistema en emulsión o microemulsión, dependiendo de la cantidad de etanol en la mezcla de reacción. El tamaño de partícula se incrementa cuando la proporción de etanol aumenta (Tabla 2), debido a que el número de partículas disminuye, al existir menos agua disponible para la formación de las partículas, con lo cual el monómero disuelto en el etanol, comenzara a emigrar hacia las partículas formadas, incrementando la adición de monómero en las redes formadas en las partículas disponibles y por consecuencia el tamaño de los polímeros formados se incrementa, este fenómeno ha sido reportado cuando se sintetizan partículas en microemulsión ${ }^{[17,18]}$. En

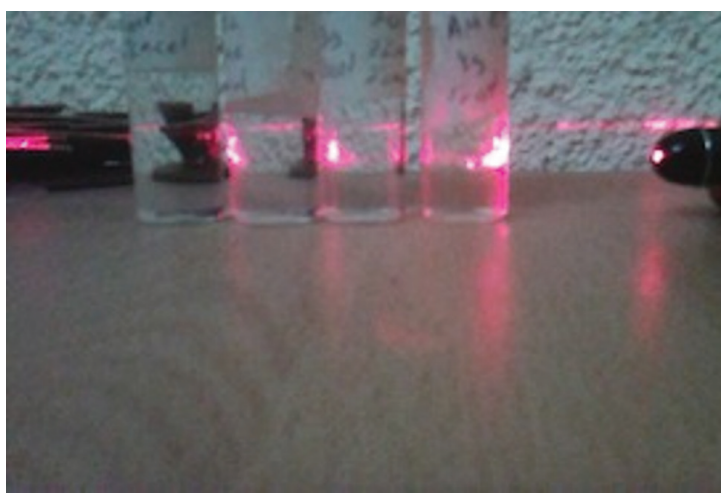

Figura 4. Se muestra la dispersión de luz en la solución de las partículas.

la Figura 4 se puede observar la dispersión de la luz en las soluciones preparadas al redispersar las partículas en agua bidestilada, cuando aparecen estas líneas continuas de luz, se puede establecer como un indicador cualitativo del tamaño nanométrico de los polímeros sintetizados ${ }^{[19]}$.

\section{Conclusiones}

En el presente trabajo se llevó acabo la síntesis de hidrogeles de acrilamida en soluciones acuosas de etanol. Se pudo observar que al variar la composición del 
etanol en la mezcla inicial de reacción se obtenían desde macrogeles hasta nanogeles. Los macrogeles modifican su estructura debido a la presencia de etanol en la solución, incrementando su capacidad de absorber agua conforme se incrementa el contenido de etanol, obteniéndose un gel heterogéneo. Así mismo la velocidad de absorber agua se ve incrementada por la presencia de estos huecos formados en las zonas ricas en etanol que se forman durante le proceso de polimerización. Se observo que cuando la composición del etanol sobrepasa el $15 \%$, se deja de formar la red obteniéndose nanogeles, con tamaños de partícula que van desde los $71 \mathrm{~nm}$ y hasta los $463 \mathrm{~nm}$.

\section{Referencias}

1. Pedley, D. G.; Skelly, P. J. \& Tighe, B. J. - Brit Polym. J., 12, p.99 (1980). http://dx.doi.org/10.1002/pi.4980120306.

2. Lee, P. I. - J. Control. Release., 2, p.277 (1985). http://dx.doi. org/10.1016/0168-3659(85)90051-3.

3. Ratner, B. D. \& Hoffman, A. S. - "Syntetic hydrogels for biomedical aplications", in: Hydrogels for medical and related applications, cap.1, Joseph D. Andrade (ed.), ACS Symposium Series, Washington (1976). http://dx.doi.org/10.1021/bk1976-0031.ch001.

4. Abrahams, R. A. \& Ronel, S. - Polym. Prepr., 16, p.535 (1975).

5. Brazel, C. S. \& Peppas, N. A. - J. Contr. Rel., 39, p.57 (1996). http://dx.doi.org/10.1016/0168-3659(95)00134-4.

6. Yuk, S. H. \& Bae, Y. H. - Crit. Rev. Ther. Drug Carrier Syst., 16, p.385 (1999). http://dx.doi.org/10.1615/ CritRevTherDrugCarrierSyst.v16.i4.20.

7. Shibayama, M. \& Tanaka, T. - "Volume phase transition and related phenomena of polymer gels", in: Responsive gels: volume transitions I, cap.1, K. Dusek (ed.), Springer-Verlag Heidelberg, New York (1993). (Advances in Polymer Science, Vol. 109). http://dx.doi.org/10.1007/3-540-56791-7_1.

8. Gehrke, S. H. - "Synthesis, equilibrium swelling, kinetics, permeability and applications of environmentally responsive gels", in: Responsive gels: volume transitions II, cap.5, K. Dusek (ed.), Springer-Verlag Heidelberg, New York (1993).
(Advances in Polymer Science, Vol. 110). http://dx.doi. org/10.1007/BFb0021130.

9. Tanaka, T. - Phys. Rev. Lett., 40, p.820 (1978). http://dx.doi. org/10.1103/PhysRevLett.40.820.

10. Hirasa, O. \& Ito S. - “IUPAC, International Symposium”, p.477 (1989).

11. Nuño-Donlucas, S. M.; Sánchez-Díaz, J. C.; Rabelero, M.; Cortés-Ortega, J.; Luhrs-Olmos, C. C.; Fernández-Escamilla, V. V.; Mendizábal, E. \& Puig, J. E. - J. Colloid Interface Sci., 270, p.94 (2004). http://dx.doi.org/10.1016/j.jcis.2003.07.045. PMid:14693139

12. Matzelle, T. R.; Geuskens, G. \& Kruse, N. - Macromolecules., 36, p.2926 (2003). http://dx.doi.org/10.1021/ma021719p.

13. Huang, J. \& Wu, X. Y. - J. Polym. Sci., 37, p.2667 (1999). http://dx.doi.org/10.1002/(SICI)10990518(19990715)37:14<2667::AID-POLA42>3.0.CO;2-J.

14. Cortes Ortega, J. A. \& Morales Borges, R. - Journal of Materials Science and Engineering, 4, p.42 (2010).

15. Gemeinhart, R. A.; Park, H. \& Park, K. - Polym. Adv. Technol., 11, p.617 (2000). http://dx.doi.org/10.1002/10991581(200008/12)11:8/12<617::AID-PAT12>3.0.CO;2-L.

16. Ruiz Pérez, U. M. - "Síntesis y purificación de hidrogeles microestructurados de acrilamida", Tesis de licenciatura en Ingeniería química, Universidad de Guadalajara, México (2012).

17. Machado, F.; Lima, E. \& Pinto, J. C. - Polímeros., 17, p.166 (2007). http://dx.doi.org/10.1590/S010414282007000200016.

18. Raberelo M. - "Látex con alto contenido de sólidos sintetizados mediante adicción continua", Tesis de Maestría, Universidad de Guadalajara, México (1996).

19. Puig, L. J.; Sanchez-Diaz, J. C.; Villacampa, M.; Mendizábal, E.; Puig, J. E.; Aguiar, A. \& Katime, I. - J. of Coll. and Inter. Sci., 235, p.278 (2001). http://dx.doi.org/10.1006/ jcis.2000.7356.

Enviado: Feb. 04, 2014 Reenviado: Abr. 08, 2014 Aceptado: Jun. 09, 2014 